\title{
Ácaros predadores (Acari) em plantas nativas e cultivadas do Estado do Rio Grande do Sul, Brasil
}

\author{
Noeli Juarez Ferla ${ }^{1}$ \\ Gilberto José de Moraes ${ }^{2}$
}

\begin{abstract}
Predators mites (Acari) in native and cultivated plants of the State of Rio Grande do Sul, Brazil. This research was carried out in twenty counties of the following regions in the state of Rio Grande do Sul: Plain, Central Depression, Plateau and Coast Plain to find out the diversity of mite predators in these places. Forty-six vegetable species were sampled, thirty species of mites of the families Anystidae, Ascidae, Cheyletidae, Cunaxidae, Phytoseiidae and Stigmaeidae were met. The Phytoseiidae were the mite that presented the greatest diversity, being present in the majority of the sample plants. Most of the Phytoseiidae that were met belong to five species of the Euseius Wainstein, 1962 genus, the second genus of this family was Iphiseiodes DeLeon, 1966, with just one species. The Stigmaeidae come up as second family in number but fewer than Phytoseiidae. In this family, the most common mite belong to the Agistemus Sumers, 1960 genus. The biggest of the mites species ( 13 species), was met in Morus spp. (Moraceae) and Tabebuia spp. (Bignoniaceae); Phaseolus vulgaris (Papilionaceae); only one species of the mite was met in Campomanesia spp. (Myrtaceae), Phaseolus vulgaris (Papilionaceae) and Rosa spp. (Rosaceae). In Alamanda spp.(Apocinaceae), Ficus spp. (Moraceae), Jacaranda mimosifolia (Bignoniaceae) and Solanum spp. (Solanaceae) were met mites predators. A dichotomic key is presented to separate the families, genus and species of the mites.

KEY WORDS. Acari, Phytoseiidae, mites, diversity of mite, Rio Grande do Sul
\end{abstract}

Ácaros predadores são freqüentemente encontrados associados a ácaros fitófagos na vegetação nativa, em culturas anuais e perenes. Os ácaros da família Phytoseiidae receberam maior atenção a partir dos anos cinqüenta, quando algumas espécies foram consideradas importantes agentes de controle de ácaros da família Tetranychidae em agroecossistemas (MORAES et al. 1986). Além dos fitoseídeos, outras famílias apresentam ácaros predadores de tetraniquídeos, destacando-se a família Stigmaeidae (LAING \& KNOP 1982). Outros grupos, apesar de algumas vezes serem reportados como importantes, parecem apresentar menor efeito sobre os tetraniquídeos (SANTOS \& LAING 1985). Os únicos relatos sobre ácaros predadores no Estado do Rio Grande do Sul são os trabalhos de LORENZATO et al. (1986), Lorenzato (1987), LORENZATO \& SECCHi (1993) e FerLa \& Moraes (1998), todos ligados à cultura da macieira.

O objetivo deste trabalho foi conhecer a diversidade de ácaros predadores plantícolas presentes em plantas nativas ou introduzidas no Estado do Rio Grande do Sul.

1) Museu de Ciências Naturais, UNIVATES Centro Universitário. Caixa Postal 155, 95900-000 Lajeado, Rio Grande do Sul. E-mail: njferla@fates.tche.br

2) Departamento de Entomologia, Fitopatologia e Zoologia Agrícola, Escola Superior de Agricultura "Luiz de Queiroz". Caixa Postal 9, 13418-900 Piracicaba, São Paulo.

Bolsista do CNPq.

Revta bras. Zool. 19 (4): 1011 - 1031, 2002 


\section{MATERIAL E MÉTODOS}

Foram realizadas coletas mensais em plantas nativas ou introduzidas, em janeiro, fevereiro e abril de 1994, em 20 municípios das Regiões da Campanha, Depressão Central, Planalto e Planície Costeira.

Em cada ponto de coleta, as cinco espécies de plantas visualmente mais comuns foram tomadas. $\mathrm{O}$ tempo de coleta foi padronizado em trinta minutos em cada espécie escolhida, examinando-se as folhas e retirando-se os ácaros com pincel de cerdas finas, com o auxílio de lupa, e introduzindo-os em frascos com álcool $70 \%$ para posterior montagem e identificação. Todas as coletas foram feitas pelo primeiro autor deste trabalho.

Para os fitoseídeos, utilizou-se o sistema de classificação genérico proposto por CHANT \& MCMURTRY (1994). As famílias e o nome científico das espécies vegetais foram identificadas com base no trabalho de SCHULTZ (1975).

Espécimes representantes de cada uma das espécies encontradas foram depositados na Coleção de Referência de Ácaros do Setor de Zoologia do Departamento de Entomologia, Fitopatologia e Zoologia Agrícola da Escola Superior de Agricultura da Universidade de São Paulo (ESALQ-USP), em Piracicaba, São Paulo.

\section{RESULTADOS}

Foi encontrado um total de 1.380 ácaros pertencentes às famílias Anystidae, Ascidae, Cheyletidae, Cunaxidae, Phytoseiidae e Stigmaeidae em 46 espécie de plantas analisadas. Trinta espécies de ácaros foram identificadas, 23 das quais pertencem à família Phytoseiidae.

São apresentadas, a seguir, as famílias acarinas e as plantas sobre as quais as espécies acarinas foram encontradas.

Anystidae Oudemans, 1902

Walzia sp.

Espécimes examinados: Santa Cruz do Sul: Cordia trichotoma, 15-IV-94; Morus sp., 20-I-94.

Ascidae Voigts \& Oudemans, 1905

Asca sp.

Espécimes examinados: Bom Retiro do Sul: Morus sp., 28-III-94; Guaporé: Rollinia sp., 06-II-94; Passo Fundo: Schinus sp., 18-II-94.

\section{Cheyletidae Leach, 1815 \\ Hemicheyletia sp.}

Espécimes examinados: Santa Cruz do Sul: Morus sp., 20-I-94.

Revta bras. Zool. 19 (4): 1011 - 1031, 2002 


\section{Cunaxidae Thor, 1902 Cunaxoides sp.}

Espécimes examinados: Guaporé: Bauhinia sp., 28-III-94; Sarandi: Citrus limon, 21-IV-94; Vera Cruz: Prunus persica, 15-II-94; Taquari: Senna sp., 10-I-94.

\section{Cunaxasp.}

Espécimes examinados: Pelotas: Morus sp., 03-IV-94; Taquari: Psidium guajava, 06-II-94.

\section{Phytoseiidae Berlese, 1913 Amblyseius (Amblyseius) chiapensis DeLeon, 1961}

Amblyseius chiapensis DeLeon, 1961: 85; DeLeon, 1962a: 175; Denmark \& Muma, 1973: 247; Denmark \& Muma, 1975: 286; Moraes \& Oliveira, 1982: 18; McMurtry, 1983: 250; Moraes \& Mesa, 1988:

72; Denmark \& Muma, 1989: 1994; McMurtry \& Moraes, 1989: 185.

Amblyseius triplaris DeLeon, 1967: 25 (sinonímia de acordo com Denmark \& Muma, 1989: 94).

Espécimes examinados: Arroio do Meio: Luehea divaricata, 28-III-94; Camaquã: Psidium guajava, 03-II-94; Carazinho: L. divaricata, 29-IV-94; Encantado: Inga sp., 28-III-94; Guaíba: P. guajava, 03-II-94; Tabebuia sp. 02-IV-94; Santa Cruz do Sul: Hibiscus rosa-sinensis L., 15-II-94; Dahlia sp., 15-II-94; Santa Maria: Inga sp., 10-IV-94; P. guajava, 10-IV-94; Sarandi: L. divaricata, 21-IV-94; Tapes: Eriobothrya japonica, 01-IV-94; Vera Cruz: Mangifera indica, 15-II-94.

Registros prévios no Brasil - Bahia, Ceará, Maranhão, Pernambuco, Rio Grande do Sul, Santa Catarina e São Paulo (Moraes et al. 1986; Moraes et al. 1993; Lorenzato \& SeCCHI 1993; Ferla \& Moraes 1998).

\section{Amblyseius (Amblyseius) herbicolus (Chant, 1959)}

Typhlodromus (Amblyseius) herbicolus Chant, 1959: 84;

Amblyseius herbicolus; Daneshvar \& Denmark, 1982: 5; McMurtry \& Moraes, 1984: 34; Denmark \& Muma, 1989: 59.

Amblyseius impactus Chaudhri, 1968: 553 (sinonímia de acordo com Daneshvar \& Denmark, 1982: 5). Amblyseius deleoni Muma \& Denmark, 1970: 68 in Muma et al., 1970: 68 (sinonímia de acordo com

Daneshvar \& Denmark, 1982: 5).

Espécimes examinados: Carazinho: Anemopaegma sp., 21-I-94; Cordia trichotoma, 18-II-94; Bauhinia sp., 21-IV-94; Guaporé: Aleurites fordii, 13-I-94.

Registros prévios no Brasil - São Paulo e Pernambuco (MORAES et al. 1986).

Iphiseiodes zuluagai Denmark \& Muma, 1972

Iphiseiodes zuluagai Denmark \& Muma, 1972: 29; Aponte \& McMurtry, 1995: 165; Kreiter \& Moraes, 1997: 377; Feres \& Moraes, 1998: 127.

Amblyseius zuluagai; Moraes \& Mesa, 1988: 79; Moraes et al., 1991: 125.

Espécimes examinados: Arroio do Meio: Mangifera indica, Passiflora sp. e Morus sp., 06-II-1994; Arroio Grande: Citrus limon, 05-I-1994; C. limon e Psidium guajava, 02-II-1994; Hibiscus rosa-sinensis, 03-IV-1994; Bom Retiro do Sul: 
Citrus sinensis, 10-I-1994; C. limon, P. guajava, Phytolacca dioica e Eriobothrya japonica, 06-II-1994; Hovenia dulcis e P. guajava, 28-III-1994; Camaquã: C. limon, 05-I-1994; Jasminum sp., E. japonica, L. divaricata, P. guajava e Passiflora sp., 02-II-1994; H. rosa-sinensis, Prunus persica e Persea gratissima, 03-IV-1994; Encantado: Psidium cattleyanum, 10-I-1994; H. rosa-sinensis, M. indica e Actinidia deliciosa, 06-II-1994; Persea gratissima e H. dulcis, 28-III-1994; Guaíba: P. guajava, 15-I-1994; Manihot esculenta e $P$. guajava, 02-II-1994; $C$. sinensis e Tabebuia sp., 03-IV-1994; Guaporé: Ilex paraguaiensis, 06-II-1994; Mata: P. gratissima e Hydrangea macrophylla, 10-II-1994; C. limon, 08-IV-1994; Santa Cruz do Sul: H. rosa-sinensis,15-II-1994; Morus sp. e Rollinia sp., 15-IV-1994; Santa Maria: Ricinus communis, 15-I-1994; Morus sp., 10-II-1994; P. guajava, 08-IV-1994; Santiago: Morus sp., 15-I-1994; C. limon e P. persica, 10-II-1994; $P$. persica e Diospyros kaki, 08-IV-1994; Tapes: C. limon e P. guajava, 05-I-1994; Inga sp. e $C$. sinensis, 02-II-1994; Taquari: $M$. indica e $C$. sinensis, 10-I-1994; $P$. guajava e Morus sp., 06-II-1994; Vera Cruz: P. guajava e Morus sp., 15-II-1994; C. sinensis, Morus sp. e C. limon,15-IV-1994.

Registros prévios no Brasil - Bahia, Maranhão, Pernambuco, Rio Grande do Sul e São Paulo (MORAES et al. 1986, 1993; Feres \& MORAes 1998; Ferla \& MORAES 1998).

\section{Amblyseius (Typhlodromips) mangleae (DeLeon, 1967)}

Typhlodromips mangleae DeLeon, 1967: 28.

Amblyseius mangleae; Moraes \& Mesa, 1988: 75; Moraes et al., 1991: 124.

Espécimes examinados: Guaporé: Morus sp., 06-II-94; Pelotas: Luehea divaricata, 02-II-94; Vera Cruz: Morus sp., 15-II-94.

Registros prévios: Brasil - São Paulo e Rio Grande do Sul (MORAES et al. 1986; FERLA \& MORAES 1998).

\section{Amblyseius (Typhlodromips) aciculus DeLeon, 1967}

Amblyseius aciculus DeLeon, 1967: 28; Moraes et al., 1991: 122.

Espécimes examinados: Pelotas: Luehea divaricata, 02-II-94.

Observação: esta é a primeira constatação desta espécie no Brasil.

\section{Amblyseius (Typhlodromalus) aripo (DeLeon, 1967)}

Typhlodromalus aripo DeLeon, 1967: 21; Denmark \& Muma, 1973: 257.

Amblyseius aripo; Moraes \& McMurtry, 1983: 132; Moraes \& Mesa, 1988: 73; Feres \& Moraes, 1998: 126.

Espécimes examinados: Mata: Ipomoea sp., 15-IV-94; Passo Fundo: Ilex paraguaiensis, 15-IV-94; Santa Cruz do Sul: Dahlia sp., 5-II-94; Santa Maria: Manihot esculenta, 15-IV-94.

Registros prévios: Brasil - Alagoas, Bahia, Ceará, Maranhão, Pará, Paraíba, Pernambuco, Piauí, Rio Grande do Norte, Rio Grande do Sul, Sergipe e São Paulo (Moraes et al. 1986, 1993; Feres \& MORAES 1998; Ferla \& MORAES 1998). 


\section{Amblyseius (Typhlodromalus) rapax DeLeon, 1965}

Amblyseius rapax De Leon 1965a: 23; DeLeon, 1967: 20; Moraes et al. 1994: 213.

Espécimes examinados: Guaíba: Tabebuia sp., 03-IV-94; Santa Cruz do Sul: Mangifera indica, 20-I-94; Vera Cruz: Morus sp., 20-I-94; Morus sp., 15-II-94; Diospyros kaki, 15-IV-94,

Observação: esta é a primeira constatação desta espécie no Brasil.

\section{Euseius alatus DeLeon, 1966}

Euseius alatus DeLeon, 1966: 87; Denmark \& Muma, 1973: 262; Moraes \& McMurtry, 1983: 137; Feres \& Moraes, 1998: 127.

Euseius paraguayensis Denmark \& Muma, 1970: 224 (sinonímia de acordo com Moraes \& McMurtry, 1983: 137).

Espécimes examinados: Arroio Grande: Platanus acerifolia, 03-IV-94; Bom Retiro do Sul: Hibiscus rosa-sinensis, 10-I-94; Phytolacca dioica, 06-II-94; Morus sp., 28-III-94; Camaquã: Citrus limon, 05-I-94; Candelária: Rollinia sp., 20-I-94; H. rosa-sinensis, 15-II-94; Cordia trichotoma, 15-IV-94; Carazinho: Persea gratissima e Morus sp., 23-I-94; Morus sp., 18-II-94; Luehea divaricata e Ilex paraguaiensis, 21-IV-94; Encantado: Morus sp. 19-I-94; Guaporé: Rollinia sp., Psidium guajava e I. paraguaiensis, 06-II-94; Aleurites fordii, Morus sp. e P. acerifolia, 28-III-94; Mata: Diospyros kaki, 15-I-94; Caria ilinoensis, 10-II-94; Hovenia dulcis, 08-IV-94; Passo Fundo: Citrus sinensis, Prunus persica e D. kaki, 23-I-94; Rollinia sp., D. kaki e Schinus sp.; 18-II-94; P. acerifolia e Inga sp., 21-IV-94; Pelotas: Chorisia speciosa, 05-I-94; H. rosa-sinensis, 02-II-94; P dioica, 03-IV-94; Santa Maria: Tabebuia sp., Morus sp. e P. dioica, 15-I-94; Morus sp., 10-II-94; P. guajava, 08-IV-94; Santa Cruz do Sul: H. rosa-sinensis, 15-II-94; Morus sp. e P. gratissima, 15-IV-94; Sarandi: I. paraguaiensis, 23-I-94; Bauhinia sp. e L. divaricata, 21-IV-94; Seberi: Morus sp., Rollinia sp. e P. persica, 23-I-94; Malus domestica, P. guajava e D. kaki, 18-II-94; P. guajava, P. acerifolia, Ocotea sp. e Eriobothrya japonica, 21-IV-94; Tapes: Morus sp. e Senna sp., 03-IV-94; Taquari: P. gratissima, 28-III-94; Vera Cruz: P. persica, 20-II-94;. P. guajava, 15-II-94; D. kaki, 15-IV-94.

Registros prévios no Brasil - Bahia, Ceará, Maranhão, Mato Grosso do Sul, Minas Gerais, Paraíba, Pernambuco e Rio Grande do Sul (MORAES et al. 1986; Feres \& Moraes 1998; FERLA \& MORAES 1998).

\section{Euseius brazilli (El-Benhawy, 1975)}

Amblyseius brazilli El-Benhawy, 1975: 574.

Espécimes examinados: Arroio do Meio: Diospyros kaki, Haemanthus coccineus, Psidium cattleyanum, Morus sp. Phaseolus vulgaris e Bougainvillea sp., 10-I-94; Morus sp., D. kaki, Citrus limon e Mangifera indica, 06-II-94; Hovenia dulcis 28-III-94; Arroio Grande: Psidium guajava, Prunus persica e Persea gratissima, 05-I-94; P. guajava, 02-II-94; Platanus acerifolia, 03-IV-94; Bom Retiro do Sul: Bauhinia sp. e Phytolacca dioica, 06-II-94; H. dulcis e Morus sp, 28-III-94; Camaquã: Inga sp., P. guajava, C. limon e $P$. dioica, 05-I-94; Morus sp. e $P$. 
guajava, 02-II-94; Inga sp., Luehea divaricata e P. gratissima, 03-IV-94; Candelária: Hibiscus rosa-sinensis e Rosa sp.,15-II-94; Cordia trichotoma e Morus sp., 15-IV-94; Encantado: H. rosa-sinensis, 10-I-94; Guaíba: P. gratissima, Morus sp., Ocotea sp. e P. guajava, 05-I-94; P. gratissima, H. rosa-sinensis, Manihot esculenta e Chorisia speciosa, 02-II-94; $P$. dioica, $P$. gratissima e Inga sp., 03-IV-94; Guaporé: H. dulcis, P. guajava e M. indica, 10-I-94; Ocotea sp., H. dulcis e Rollinia sp., 06-II-94; Aleurites fordii, $D$. kaki e $P$. dioica, 28-II-94; Mata: $P$. gratissima e D. kaki, 15-I-94; Erytrina crista-galli e $P$. gratissima, 11-II-94; Ipomoea sp. e $H$. dulcis, 08-IV-94; Passo Fundo: Rollinia sp. e Hydrangea macrophylla, 23-I-94; Rollinia sp., Schinus sp. e Eriobothrya japonica, 18-II-94; Inga sp., P. acerifolia, Ilex paraguaiensis e H. macrophylla, 21-IV-94; Pelotas: Morus sp., D. kaki, Malus domestica, C. speciosa e H. macrophylla, 05-I-94; P. gratissima e H. rosa-sinensis e P. guajava, 02-II-94; P. persica, $P$. dioica, $P$. cattleyanum e H. macrophylla, 03-IV-94; Santa Cruz do Sul: I. paraguaiensis, D. kaki e Citrus sinensis, 20-I-94; D. kaki, 15-II-94; P. gratissima, P. guajava e C. trichotoma, 15-IV-94; Santa Maria: P. gratissima, C. speciosa e P. dioica, 20-I-94; Bauhinia sp. e P. acerifolia, 10-II-94; Ocotea sp., 08-IV-94; Santiago: Rosa sp., Morus sp., P. dioica e P. persica, 15-I-94; Sarandi: Ricinus communis, 23-I-94; Ocotea sp. e L. divaricata, 18-II-94; Seberi: $P$. gratissima, 21-IV-94; Taquari: $P$. gratissima e $H$. macrophylla, 10-I-94; $H$. macrophylla, 06-II-94; $H$. rosa-sinensis, Rollinia sp., $P$. gratissima e $P$. cattleyanum, 28-III-94; Vera Cruz: P. gratissima, 15-II-94; D. kaki 15-IV-94.

Registros prévios no Brasil - São Paulo e Rio Grande do Sul (MORAES et al. 1986; Feres \& MORAES 1998; Ferla \& MORAES 1998).

\section{Euseius citrifolius Denmark \& Muma, 1970}

Euseius citrifolius Denmark \& Muma, 1970: 222; Moraes \& McMurtry, 1983: 138; Feres \& Moraes, 1998: 127

Espécimes examinados: Arroio do Meio: Mangifera indica, 06-II-94; Bauhinia sp., 28-III-94; Bom Retiro do Sul: Persea gratisssima, 10-I-94; Psidium guajava, 06-II-94; $P$. gratissima e $P$. guajava, 28-III-94; Candelária: $P$. guajava, Rollinia sp., Prunus persica, Diospyros kaki e Inga sp., 20-I-94; Senna sp., 15-II-94; Carazinho: Luehea divaricata, 21-IV-94; Encantado: Chorisisa speciosa, 06-II-94; Mata: Senna sp. e P. gratissima, 15-I-94; P. gratissima, 10-II-94;, P. gratissima e D. kaki, 08-IV-94; Santa Cruz do Sul: L. divaricata, 15-IV-94; Seberi: P. persica e Phytolacca dioica, 23-I-94; Morus sp., 18-II-94; P. gratissima e Ocotea sp., 21-IV-94; Taquari: P. gratissima, 10-I-94.

Registros prévios no Brasil - Bahia, Ceará, Maranhão, Mato Grosso do Sul, Minas Gerais, Paraíba, Pernambuco, Piauí, Rio Grande do Sul e São Paulo (MORAES et al. 1986; Feres \& MORAES 1998; Ferla \& MORAES 1998).

\section{Euseius concordis (Chant, 1959)}

Typhlodromus (Amblyseius) concordis Chant, 1959: 69.

Amblyseius concordis; Chant \& Baker, 1965: 22

Euseius concordis; Denmark \& Muma, 1973: 264; Moraes \& Oliveira, 1982: 317; Feres \& Moraes, 1998 : 127. 
Euseius flechtmanni Denmark \& Muma, 1970: 223; Denmark \& Muma, 1973: 261 (sinonímia de acordo com Moraes et al. 1982: 18).

Espécimes examinados: Arroio do Meio: Lycopersicon esculentum, Hibiscus rosa-sinensis, Bougainvillea sp. e Psidium cattleyanum, 10-I-94; Rollinia sp. e Passiflora sp., 06-II-94; Luehea divaricata e Bauhinia sp., 28-III-94; Bom Retiro do Sul: H. rosa-sinensis, Citrus sinensis e Psidium guajava; 10-I-94; P. guajava e Eriobothrya japonica, 06-II-94; Tabebuia sp., 28-III-94; Camaquã: Citrus limon, 05-I-94; Candelária: Prunus persica, Morus sp. e H. rosa-sinensis; Carazinho: Persea gratissima, 23-I-94; L. divaricata, 18-II-94; Rollinia sp. e L. divaricata, 21-IV-94; Encantado: Bauhinia sp., Rollinia sp., Manihot esculenta e Chorisisa speciosa, 10-I-94; C. sinensis, P. guajava, Phytolacca dioica e Actinidia deliciosa, 06-II-94; Inga sp., P. guajava e P. gratissima, 28-III-94; Guaporé: Hydrangea macrophylla, 10-I-94; Santa Cruz do Sul: $L$. divaricata, 15-IV-94; Santa Maria: $P$. dioica e Ricinus communis, 15-I-94; Eriobothrya japonica, 10-II-94; Santiago: Passiflora sp., P. gratissima e $C$. limon, 10-II-94; H. rosa-sinensis, 08-IV-94; Sarandi: P. guajava, 23-I-94; Ocotea sp., 18-II-94; Seberi: Ilex paraguaiensis e P. dioica, 23-I-94; I. paraguaiensis, Morus sp. e P. dioica, 18-II-94; Prunus persica, P. gratissima, Ocotea sp. e P. guajava, 21-IV-94; Vera Cruz: $P$. persica e $P$. guajava, 18-II-94.

Registros prévios no Brasil - Bahia, Ceará, Maranhão, Mato Grosso do Sul, Paraíba, Pernambuco, Piauí, Rio Grande do Sul e São Paulo (MORAES et al. 1986; FERES \& MORAES 1998; FERLa \& MORAES 1998).

\section{Euseius sibelius (DeLeon, 1962)}

Amblyseius (Typhlodromalus) sibelius DeLeon, 1962b: 21.

Euseius sibelius; Muma et al., 1970: 98; Feres \& Moraes, 1998: 128.

Euseius subalatus DeLeon, 1965a: 127; (sinonímia de acordo com Muma et al., 1970: 35)

Espécimes examinados: Bom Retiro do Sul: Tabebuia sp., 10-I-94; Sarandi: Diospyros kaki, 18-II-94.

Registros prévios: Brasil - Bahia, Mato Grosso do Sul, Paraíba, Pernambuco, Piauí, Rio Grande do Sul e São Paulo (MORAES et al. 1986; Feres \& MORAES 1998; FERLA \& MORAES 1998).

\section{Euseius inouei Ehara \& Moraes, 1998}

Euseius inouei Ehara \& Moraes, 1998: 59.

Espécimes examinados: Mata: Citrus sinensis, 10-I-94; Passo Fundo: Senna sp., 23-II-94.

Observação: esta é a primeira constatação desta espécie no Brasil.

\section{Neoseiulus californicus (McGregor, 1954)}

Typhlodromus californicus McGregor, 1954: 89.

Amblyseius californicus; Schuster \& Pritchard, 1963: 271; McMurtry, 1977: 21.

Neoseiulus californicus; McMurtry \& Moraes, 1989: 181.

Typhlodromus chilenensis Dosse, 1958: 3 (sinonímia de acordo com Athias-Henriot, 1977: 62 e El-Benhawy, 1979: 113). 
Espécimes examinados: Carazinho: Manihot esculenta, 18-II-94.

Registros prévios no Brasil - Rio Grande do Sul, Santa Catarina e São Paulo (Moraes et al. 1986; Ferla \& MORAES 1998).

\section{Neoseiulus tunus (DeLeon, 1967)}

Typhlodromips tunus DeLeon, 1967: 29; Denmark \& Muma, 1973: 253.

Amblyseius tunus; Feres \& Moraes, 1998: 126.

Espécimes examinados: Bom Retiro do Sul: Tabebuia sp. e Rollinia sp., 28-II-94; Camaquã: Morus sp., 02-II-94; Hibiscus rosa-sinensis, 03-IV-94; Carazinho: Anemopaegma sp., Morus sp. e Hovenia dulcis, 23-I-94; Luehea divaricata e Cordia trichotoma, 18-II-94; Encantado: Bauhinia sp., 10-I-94; H. dulcis, 28-III94; Guaíba: H. rosa-sinensis, Senna sp. e Psidium guajava, 02-II-94; Tabebuia sp., 03-IV-94; Guaporé: Bauhinia sp., 28-III-94; Mata: P. guajava, 15-I-94; Tabebuia sp., 10-II-94; Passo Fundo: Rollinia sp. e Tabebuia sp.; 21-IV-94; Pelotas: Ficus . sp. e P. guajava, 02-II-94; Santa Cruz do Sul: Senna sp., 20-I-94; Santa Maria: Manihot esculenta, Bauhinia sp., Platanus acerifolia e Tabebuia sp., 10-II-94; Sarandi: Persea gratissima, 18-II-94.

Registros prévios no Brasil - Rio Grande do Sul, Santa Catarina e São Paulo (MORAES et al. 1986; Feres \& MORAES 1998; Ferla \& MORAES 1998).

\section{Paraamblyseius metapodalis (El-Benhawy, 1984)}

Amblyseius metapodalis El-Benhawy, 1984: 132.

Espécimes examinados: Arroio Grande: Citrus limon, 05-I-94; Hibiscus rosa-sinensis, 03-IV-94; Bom Retiro do Sul: Tabebuia sp., 10-I-94; Psidium guajava, Inga sp. e C. limon, 06-II-94; Camaquã: C. limon, 05-I-94; Jasminum sp., 02-II-94; Guaíba: Tabebuia sp., 03-IV-94; Pelotas: P. guajava e Ficus sp., 02-II-94; Santa Cruz do Sul: Diospyros kaki, 15-II-94; Cordia trichotoma, 15-IV-94; C. limon, 15-IV-94; Santiago: Passiflora sp., 10-II-94; H. rosa-sinensis, 08-IV-94; Sarandi: C. limon, 21-IV-94; Tapes: Citrus sinensis e Hovenia dulcis, 02-II-94.

Registros prévios no Brasil - Espirito Santo e Rio Grande do Sul (MORAES et al. 1986; FERLA \& MORAES 1998).

\section{Phytoseiulus macropilis (Banks, 1905)}

Laelaps macropilis Banks, 1905:139.

Phytoseiulus speyeri Evans, 1952:398 (sinonímia de acordo com Kennet, 1958: 477).

Phytoseiulus chanti Ehara, 1966: 135 (sinonímia de acordo com Denmark \& Muma, 1973: 236).

Phytoseiulus macropilis; Schuster \& Pritchard, 1963: 279; Muma et al., 1970: 30; McMurtry, 1983: 259; Denmark \& Schicha, 1983: 31; Kreiter \& Moraes, 1997: 378.

Espécimes examinados: Carazinho: Morus sp., 23-I-94; Bom Retiro do Sul: Tabebuia sp., 10-I-94; Guaíba: Morus sp., 05-I-94; Santa Cruz do Sul: Tabebuia sp., 20-I-94; Santiago: Citrus sinensis, 15-I-94.

Registros prévios no Brasil - Bahia, Ceará, Fernando de Noronha, Minas Gerais, Pernambuco, Rio Grande do Sul, Santa Catarina e São Paulo (MoraEs et al. 1986). 


\section{Proprioseiopsis neotropicus (Ehara, 1966)}

Amblyseius neotropicus Ehara, 1966: 133; Moraes \& Mesa, 1988: 79; Moraes et al., 1991: 127.

Espécimes examinados: Santa Cruz do Sul: Citrus limon, 15-IV-94; Vera Cruz: Manihot esculenta, 18-II-94.

Registros prévios no Brasil - Bahia, São Paulo e Rio Grande do Sul (MORAES et al. 1986, 1991; FERLA \& MORAES 1998).

\section{Phytoseius guianensis DeLeon, 1965}

Phytoseius (Pennaseius) guianensis DeLeon, 1965b: 18; Denmark, 1966: 23; Denmark \& Muma, 1983: 144.

Espécimes examinados: Arroio do Meio: Diospyros kaki, 28-III-94; Carazinho: Inga sp. e Bauhinia sp., 21-IV-94; Guaíba: Tabebuia sp. e Actinidia deliciosa, 03-IV-94; Guaporé: Rollinia sp., 06-II-94; Cordia trichotoma, 28-III-94; Mata: Tabebuia sp., 10-II-94; Passo Fundo: Schinus sp., 18-II-94; Sarandi: Luehea divaricata, 18-II-94; Seberi: Bauhinia sp., 23-I-94; Taquari: Tabebuia sp., 06-II-94.

Registros prévios no Brasil - Bahia, Ceará, Paraíba, Pernambuco e Rio Grande do Sul (MORAES et al. 1986; Ferla \& MORAES 1998).

\section{Galendromus (Galendromus) annectens (DeLeon, 1958)}

Typhlodromus annectens DeLeon, 1958: 75; Chant \& Yoshida-Shaul, 1984: 1868.

Galendromus (Galendromus) annectens; Muma, 1963: 30.

Espécimes examinados: Candelária: Inga sp., 20-I-94; Carazinho: Bauhinia sp., 21-IV-94; Encantado: Diospyros kaki, 28-III-94; Guaporé, Bauhinia sp., 28-III94; Mata: D. kaki, 08-IV- 94; Santa Cruz do Sul: Bauhinia sp., 15-IV-94; Santa Maria: Tabebuia sp.,10-II-94; Sarandi: Bauhinia sp., 21-IV-94; Seberi: Malus domestica, 18-II-94; Tapes: Luehea divaricata, 02-II-94.

Registros prévios no Brasil - Pernambuco, São Paulo e Rio Grande do Sul (MORAES et al. 1986; FERES \& MORAES 1998; Ferla \& MORAES 1998).

Galendromus (Mugidromus) aff. mexicanus Muma, 1963

Espécimes examinados: Passo Fundo: Tabebuia sp.,15-II-94.

Registros prévios no Brasil: Rio Grande do Sul (LoREnZATo \& SECCHI 1987; FERLA \& MORAES 1998).

Metaseiulus (Metaseiulus) camelliae (Chant \& Yoshida-Shaul, 1983)

Typhlodromus camelliae Chant \& Yoshida-Shaul, 1983: 1057.

Espécimes examinados: Arroio Grande: Psidium guajava, 02-II-94; Mata: Carya ilinoensis, 10-II-94; Passo Fundo: Tabebuia sp. e Senna sp., 18-II-94; Santiago: Senna sp., 10-II-94; Seberi: Campomanesia sp., 21-IV-94.

Registro prévio no Brasil: Rio Grande do Sul (LORENZATO \& SECCHI 1993; FERLA \& MORAES 1998). 


\section{Typhlodromus (Anthoseius) transvaalensis (Nesbitt, 1955)}

Kampimodromus transvaalensis Nesbitt, 1951: 55.

Typhlodromus transvaalensis; Chant, 1955: 498.

Espécimes examinados: Santiago: Diospyros kaki, 08-IV-94.

Registros prévios no Brasil: São Paulo e Rio Grande do Sul (MORAES et al.1986; FERLA \& MORAES 1998).

\section{Stigmaeidae Oudemans, 1931 Agistemus sp.}

Espécimes examinados: Arroio do Meio: Citrus limon, 06-II-94; Arroio Grande: Diospyros kaki, 02-II-94; Candelária: Ficus sp., 15-IV-94; Encantado: Psidium guajava, 06-II-94; Guaporé: Ilex paraguaiensis, 06-II-94; Passo Fundo: Persea gratissima, 23-I-94; Rollinia sp., 21-IV-94; Santa Cruz do Sul: D. kaki, Hibiscus rosa-sinensis, Morus sp., 15-IV-94; Santa Maria: Morus sp., 15-I-94; Ocotea sp., 08-IV-94; Sarandi: Citrus sinensis, 21-IV-94; Seberi: P. guajava, Malus domestica,18-II-94; D. kaki, 21-IV-94; Tapes: Eriobothrya japonica, 03-IV-94; Taquari: Senna sp., 10-I-94; Vera Cruz: P. gratissima, 15-II-94.

\section{Zetzellia sp.}

Espécimes examinados: Tapes: Senna sp., 03-IV-94.

Neste estudo, os fitoseídeos foram os ácaros que apresentaram maior diversidade, estando presentes na maioria das plantas amostradas. Vinte e três espécies, pertencentes a 11 gêneros estiveram representados, sendo que três espécies de fitoseídeos são relatadas pela primeira vez no Brasil.

As espécies de ácaros predadores encontradas podem ser separadas pela seguinte chave taxonômica.

\section{Chave para a identificação de fêmeas de ácaros predadores encontrados neste estudo no Rio Grande do Sul}

1. Tarso do palpo sem apotele; tritosterno ausente; sem estigmas entre as coxas III

e IV . . Ordem Acariformes ... Subordem Actinedida . . . . . . . . . . . 2

- Tarso do palpo com apotele; tritosterno presente; estigmas localizados lateralmen-

te entre as coxas III e IV, e associados a peritrema ... Ordem Parasitiformes

.... Subordem Mesostigmata ....................... 7

2. Palpo com seta dorsal da tíbia modificada em esporão, que desloca o tarso lateral ou ventralmente (complexo "unha-dedão") . . . . . . . . . . . 3

- Palpo com complexo "unha-dedão" de forma diferente, ou esse indistinto . . . 5

3. Bases das quelíceras fundidas com o restante do gnatossoma; tarso do palpo com seta modificada robusta e em forma de foice, tarso do palpo com duas setas pectinadas; segmentos do palpo mais robustos que os das pernas... Cheyletidae ............................ Hemicheyletia 
- Bases das quelíceras totalmente separadas ou parcialmente fundidas, porém sempre independentes do restante do gnatossoma; tarso do palpo sem setas modificadas em forma de foice e tarso do palpo sem setas pectinadas; segmentos do palpo de diâmetro semelhante ao das pernas ... Stigmaeidae

4. Escudo central do histerossoma com 5 pares de setas na fêmea e 6 no macho ... Agistemus

- Escudo central do histerossoma da fêmea e do macho com 4 pares de setas ..... Zetzellia

5. Palpo com "dedão" longo e proeminente; quelíceras recurvadas distalmente; anteriormente, o dorso do propodossoma mostra um tubérculo que leva um par de setas ... Anystidae ...........................

- Palpo de comprimento aproximadamente igual ao das quelas; segmento distal raptorial; com 2 pares de discos genitais; dorsalmente, no prodossoma ocorrem dois pares de órgãos pseudo-estigmáticos bem destacados . . .

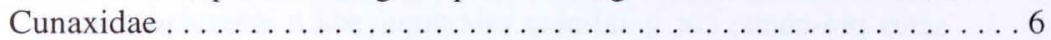

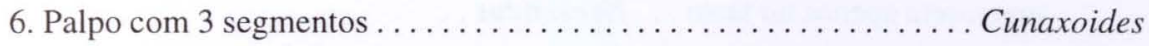

- Palpo com 5 segmentos

Cunaxa

7. Com mais de 24 pares de setas no dorso do idiossoma; setas Z4 e S5 inseridas em tubérculos póstero-laterais da região opistonotal do escudo dorsal . . . Ascidae ..........................................

- Com menos de 24 pares de setas no dorso do idiossoma; setas Z4 e S5 inseridas sobre o escudo dorsal na região opistonotal . . Phytoseiidae . . . . . . 8

8. Região podonotal do escudo dorsal com 4 pares de setas "laterais" (j3, z2, z4 e s4) ... Amblyseiinae . . . . . . . . . . . . . . . . . . . 9

- Região podonotal do escudo dorsal com 6 pares de setas "laterais" (j3, z2, z3, z4,

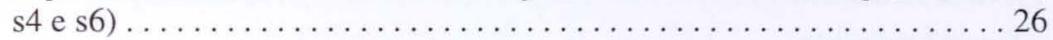

9. Setas j6 duas a três vezes mais longas que a distância entre suas bases; escudo ventrianal com um par de setas pré-anais ........ Phytoseiulus macropilis

- Setas j6 menores que duas vezes a distância entre suas bases; escudo ventrianal

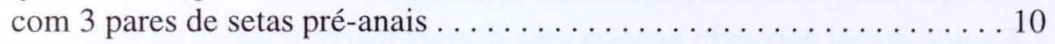

10. Setas pré-anais dispostas aproximadamente em linha transversal, com a seta JV1 afastada da margem anterior do escudo ventrianal; peritrema normalmente curto, raramente estendendo-se além da base da seta z2; dígito fixo da quelícera com 4-5 dentes, todos distais . . Euseius . . . . . . . . . . . 11

- Seta JV1 inserida próxima a margem do escudo ventrianal; peritrema normalmen-

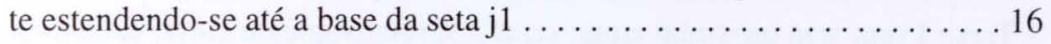

11. Macrosetas da perna IV com extremidade distal dilatada . . . . . . . . 12

- Macrosetas da perna IV com extremidade distal afilada . . . . . . . . . . 15

12. Macrosetas presentes somente na perna IV; setas $\mathrm{r} 3$ e R1 inseridas no escudo dorsal .............................. Euseius sibelius 
- Macrosetas presentes no gênu, tíbia e tarso da perna IV e no gênu da perna III; seta $\mathrm{r} 3$ inserida na membrana interescutal; setas R1 normalmente inserida na membrana interescutal ou, raramente no escudo dorsal . . . . . . . . 13

13. Escudo dorsal com poucas estrias na região antero-lateral; seta z2 menor que $\mathrm{z} 4$, que por sua vez é menor que $54 \ldots \ldots \ldots \ldots \ldots$ Euseius concordis

- Escudo dorsal totalmente reticulado; comprimentos relativos das setas z2, z4 e s4 diferentes dos citados acima ....................... 14

14. Peritrema curto, atingindo a região entre as setas $\mathrm{j} 3 \mathrm{e} \mathrm{z} 2 \ldots$. . Euseius alatus - Peritrema longo, terminando próximo à base da seta $\mathrm{j} 1$........ Euseius inouei

15. Dorso do escudo dorsal reticulado ............... Euseius citrifolius - Dorso do escudo dorsal liso . Euseius brazilli

16. Macrosetas presentes apenas na perna IV.

- Macrosetas presentes também em outras pernas

17. Escudo esternal e ventrianal pelo menos tão longos quanto largos; três pares de setas pré-anais; cor geralmete esbranquiçada a amarelada; perna IV com macroseta apenas no tarso . . Neoseiulus . . . . . . . . . . . . . 18

- Escudo ventrianal mais largo que longo; quatro pares de setas pré-anais; cor marron; maior parte do ventre coberto por escudos fortes e ornamentados; macrosetas presentes no gênu, tíbia e tarso da perna IV . ............. Paraamblyseius metapodalis

18. Setas do escudo dorsal lisas, exceto as setas Z5 que são serreadas; cálice da espermateca em forma de taça; macroseta do tarso IV com $48 \mu$ m de comprimento . .......................... Neoseiulus californicus

- Todas as setas do escudo serrilhadas; cálice da espermateca em forma de um curto sino; macroseta do tarso IV com $29 \mu \mathrm{m}$ de comprimento ... Neoseiulus tunus

19. Seta J2 ausente .................... Proprioseiopsis neotropicus

- Seta J2 presente ............................... 20

20. Escudo dorsal amplo, cobrindo quase toda a superfície superior do idiossoma, escudo esternal e ventrianal mais largos que longos; marrons; macrosetas da perna IV com extremidade distal dilatada ........... Iphiseiodes zuluagai

- Escudo dorsal deixando exposta uma distinta margem lateral da superfície dorsal do idiossoma; escudo esternal e ventrianal normalmente mais longos que largos; esbranquiçados a amarelados; macrosetas da perna IV com extremidade distal afilada . .

21. Tarso I com seta proximal ereta; setas Z5 consideravelmente maiores que a distância entre suas bases...Amblyseius (Amblyseius)............. 22

- Tarso I sem seta proximal ereta; setas Z5, relativamente curta, usualmente mais

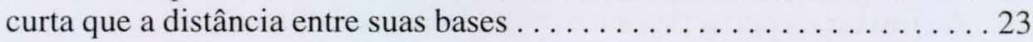

22. Cálice da espermateca em forma de taça rasa ............... chiapensis - Cálice da espermateca alongado, alargando-se próximo à vesícula . . . . . . . . . 
23. Margem posterior do escudo esternal trilobada, frequentemente muito pouco esclerotizada; escudo ventrianal com constrição lateral; setas JV1 inseridas junto à margem anterior deste escudo ... Amblyseius (Typhlodromalus). . 24

- Margem posterior do escudo esternal reta ou côncava, sempre bem visível; escudo ventrianal aproximadamente pentagonal; setas JV1 inseridas próxima à margem anterior deste escudo ... Amblyseius Typhlodromips . . . . . . 25

24. Escudo dorsal liso; setas j1, j3, s4 e Z5 muito maiores que as demais setas do escudo dorsal; macrosetas afiladas ................... (T.) rapax

- Escudo dorsal areolado; seta z2 menor que z4, que por sua vez é menor que s4; macrosetas da perna IV levemente dilatadas ............... (T.) aripo

25. Escudo dorsal liso; seta Z4 aproximadamente tão longa quanto a distância entre sua base e a base de Z5

A. (T.) aciculus

- Escudo dorsal reticulado; seta Z4 muito mais curta que a distância entre a sua base e a base de Z5 A.(T.) mangleae

26. Seta $\mathrm{r} 3$ sobre o escudo dorsal; todas as setas $\mathrm{S}$ ausentes; setas do escudo dorsal clavadas; somente a seta Z5 serrilhada ... Phytoseiinae . . .

Phytoseius guianensis

- Seta r3 no tegumento; uma ou mais setas $\mathrm{S}$ presentes . . . Typhlodrominae . . 27

27. Setas S4 e JV4 presentes; seta JV3 ausente; quase todas as setas do escudo dorsal longas, serreadas e com dilatação no ápice . . . . . . . . . . . . . . Typhlodromus (Anthoseius) transvaalensis

- Setas S4 e JV4 ausentes; seta JV3 presente; setas do escudo dorsal afilando-se progressivamente da base ao ápice . . . . . . . . . . . . . 28

28. Seta R1 presente; setas S4 presente e S2 ausente; setas R1 e s6 de comprimentos semelhantes .................. Metaseiulus (Metaseiulus) camelliae

- Seta R1 ausente; setas S4 ausente e S2 presente; setas R1 e s6 de comprimentos

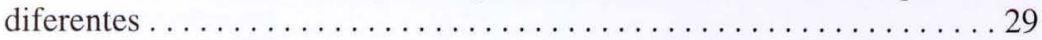

29. Peritrema estendendo-se até próximo de j1; j3 inserida mais próximo de $\mathrm{z} 2$ do que de $\mathrm{j} 1 ; \mathrm{z} 3$ inserida mais próxima da inserção de z2 que de $\mathrm{z} 4$ e bem lateral em relação à linha entre z2 e z4; setas dorsais curtas; setas s 4 e j4 muito mais curtas que a distância entre as suas inserções e as inserções das setas imediatamente após estas; base do cálice da espermateca em forma de corneta ................ Galendromus (Mugidromus) aff. mexicanus

- Peritrema curto, alcançando pouco além da base da seta r3; j3 inserida mais próximo da seta $\mathrm{j} 1$ do que da seta $\mathrm{z} 2 ; \mathrm{z} 3$ eqüidistante e em linha com $\mathrm{z} 2 \mathrm{e}$ z4; setas dorsais longas; setas s4 e j4 mais longas que a distância entre suas inserções e as inserções das setas imediatamente após estas; cálice da espermateca tubular.......... Galendromus (Galendromus) annectens

Os ácaros encontrados em cada uma das espécies de plantas amostradas são indicados na tabela I. 
Tabela I. Ácaros predadores encontrados em 20 municípios da Campanha, Depressão Central, Planalto e Planície Costeira do Estado do Rio Grande do Sul, nos meses de janeiro, fevereiro e abril de 1994.

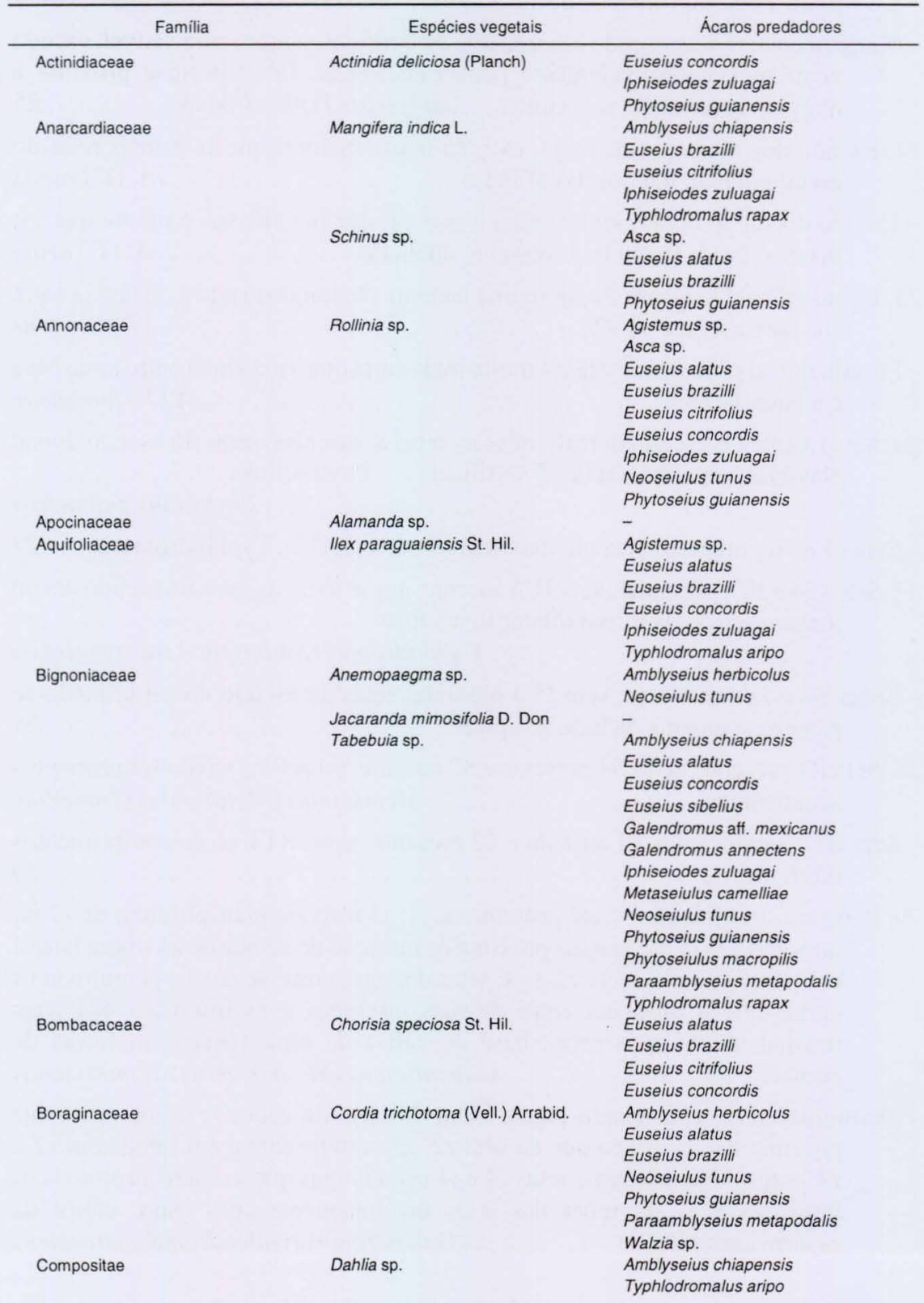


Tabela I. Continuação.

\begin{tabular}{|c|c|c|}
\hline Família & Espécies vegetais & Ácaros predadores \\
\hline Convolvulaceae & Ipomoea sp. & $\begin{array}{l}\text { Euseius brazilli } \\
\text { Typhlodromalus aripo }\end{array}$ \\
\hline Ebenaceae & Diospyros kaki L. & $\begin{array}{l}\text { Agistemus sp. } \\
\text { Euseius alatus } \\
\text { Euseius brazilli } \\
\text { Euseius citrifolius } \\
\text { Euseius sibelius } \\
\text { Galendromus annectens } \\
\text { Iphiseiodes zuluagai } \\
\text { Phytoseius guianensis } \\
\text { Paraamblyseius metapodalis } \\
\text { Typhlodromalus rapax } \\
\text { Typhlodromus transvaalensis }\end{array}$ \\
\hline Euphorbiaceae & Aleurites fordii Hemsl. & $\begin{array}{l}\text { Amblyseius herbicolus } \\
\text { Euseius alatus } \\
\text { Euseius brazilli }\end{array}$ \\
\hline & 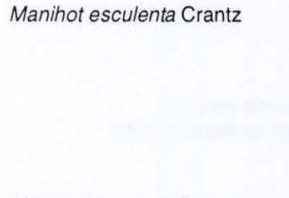 & $\begin{array}{l}\text { Euseius brazilli } \\
\text { Euseius concordis } \\
\text { Iphiseiodes zuluagai } \\
\text { Neoseiulus californicus } \\
\text { Neoseiulus tunus } \\
\text { Proprioseiopsis neotropicus } \\
\text { Typhlodromalus aripo }\end{array}$ \\
\hline & Ricinus communis $\mathrm{L}$. & $\begin{array}{l}\text { Euseius brazilli } \\
\text { Euseius concordis } \\
\text { Iphiseiodes zuluagai }\end{array}$ \\
\hline Juglandaceae & Carya ilinoensis E. Graebner & $\begin{array}{l}\text { Euseius alatus } \\
\text { Metaseiulus camelliae }\end{array}$ \\
\hline Lauraceae & $x^{2}$ & $\begin{array}{l}\text { Agistemus sp. } \\
\text { Euseius alatus } \\
\text { Euseius brazilli } \\
\text { Euseius citrifolius } \\
\text { Euseius concordis }\end{array}$ \\
\hline & Persea gratissima Gaertn. & $\begin{array}{l}\text { Agistemus sp. } \\
\text { Euseius alatus } \\
\text { Euseius brazilli } \\
\text { Euseius citrifolius } \\
\text { Euseius concordis } \\
\text { Iphiseiodes zuluagai } \\
\text { Neoseiulus tunus }\end{array}$ \\
\hline Leguminosae & $x^{2}$ & $\begin{array}{l}\text { Amblyseius herbicolus } \\
\text { Cunaxoides sp. } \\
\text { Euseius alatus } \\
\text { Euseius brazilli } \\
\text { Euseius citrifolius } \\
\text { Euseius concordis } \\
\text { Galendromus annectens } \\
\text { Neoseiulus tunus } \\
\text { Phytoseius guianensis }\end{array}$ \\
\hline & $\begin{array}{l}\text { Erythrina crista-galli L. } \\
\text { Inga sp. }\end{array}$ & $\begin{array}{l}\text { Euseius brazilli } \\
\text { Amblyseius chiapensis } \\
\text { Euseius alatus } \\
\text { Euseius brazilli } \\
\text { Euseius citrifolius } \\
\text { Euseius concordis } \\
\text { Galendromus annectens } \\
\text { Iphiseiodes zuluagai } \\
\text { Phytoseius guianensis } \\
\text { Paraamblyseius metapodalis }\end{array}$ \\
\hline
\end{tabular}

Continua

Revta bras. Zool. 19 (4): 1011 - 1031, 2002 
Tabela I. Continuação.

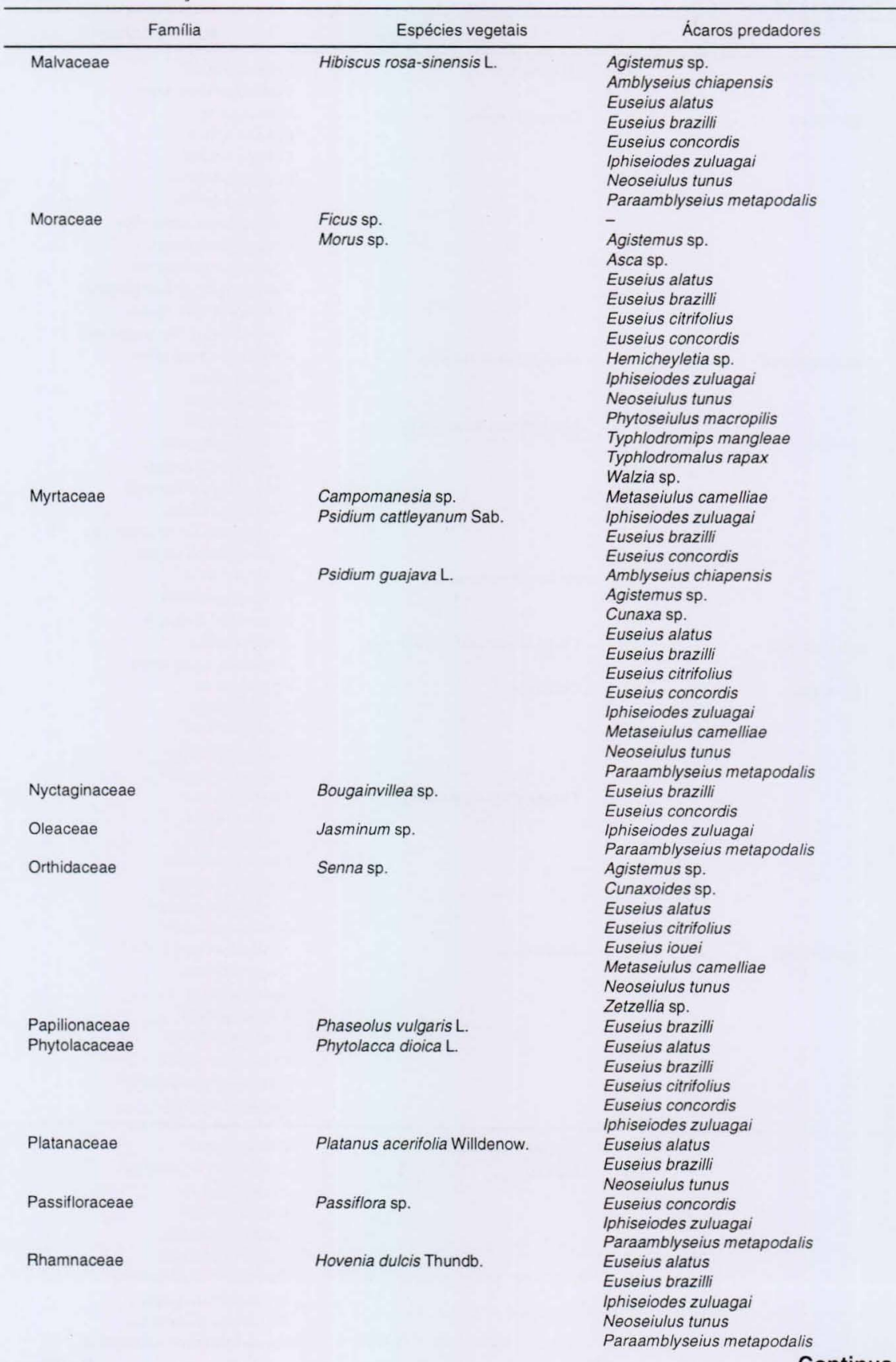


Tabela I. Continuação.

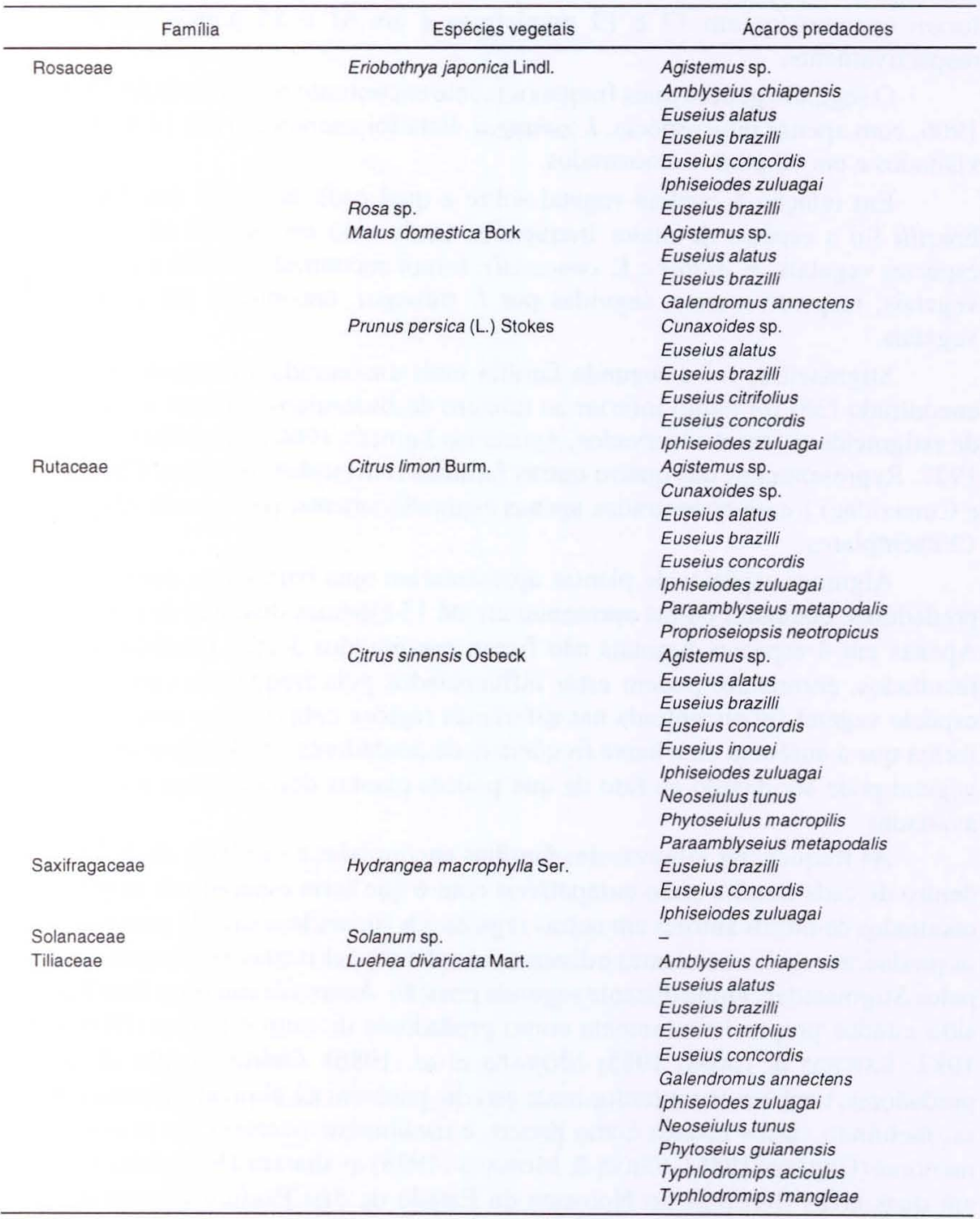

\section{DISCUSSÃO}

Phytoseiidae foi a família mais encontrada neste estudo. Dos 1380 ácaros coletados neste estudo, 1330 ácaros pertenciam a esta família. Nesta família o gênero Euseius Wainstein, 1962 apresentou maior número de espécies, sendo estas coletadas na maioria dos pontos amostrados. As espécies mais comuns, deste gênero, foram E. brazilli, E. alatus e E. concordis. E. brazilli foi encontrada em 17 dos 20 
municípios visitados e em 110 dos 300 pontos amostrados. E. alatus e E. concordis foram encontrados em 17 e 13 municípios e em 61 e 55 pontos amostrados, respectivamente.

O segundo gênero mais freqüentemente encontrado foi Iphiseiodes DeLeon, 1966, com apenas uma espécie, I. zuluagai. Esta foi encontrada em 14 municípios visitados e em 63 pontos amostrados.

Em relação à espécie vegetal sobre a qual cada ácaro foi encontrado, $E$. brazilli foi a espécie de maior frequência, tendo sido encontrada em 33 das 46 espécies vegetais. E. alatus e E. concordis, foram encontradas em 26 e 24 espécies vegetais, respectivamente, seguidas por I. zuluagai, encontrada em 23 espécies vegetais.

Stigmaeidae foi a segunda família mais encontrada, entretanto o número encontrado (38) foi muito inferior ao número de fitoseídeos. Apenas dois gêneros de estigmeídeos foram observados, Agistemus Sumers, 1960 e Zetzellia Oudemans, 1927. Representantes das quatro outras famílias (Anystidae, Ascidae, Cheyletidae e Cunaxidae) foram encontrados apenas esporadicamente, perfazendo um total de 12 exemplares.

Algumas espécies de plantas apresentaram uma baixíssima diversidade de predadores, enquanto outras apresentaram até 13 espécies distintas de predadores. Apenas em 4 espécies vegetais não foram encontrados ácaros predadores. Estes resultados, entretanto, podem estar influenciados pela freqüência com que cada espécie vegetal foi encontrada nas diferentes regiões cobertas por este estudo, de forma que a ausência ou a baixa freqüência de predadores em determinada espécie vegetal pode ser devido ao fato de que poucas plantas desta espécie tenham sido avaliadas.

As freqüências relativas das famílias encontradas e os níveis de diversidade dentro de cada família estão compatíveis com o que seria esperado de acordo com resultados de outros autores em outras regiões. Os fitoseídeos são, de maneira geral, os predadores mais abundantes e diversos em plantas cultivadas e silvestres, seguida pelos Stigmaeidae, numa distante segunda posição. Ácaros destas duas famílias tem sido citados preponderantemente como predadores de outros ácaros (HoY et al. 1982; SANTOS \& LAING 1985; MoraES et al. 1986). Outros grupos de ácaros predadores, também encontrados neste estudo, parecem ter alimentação mais diversa, incluindo vários insetos como presas, e usualmente ocorrem em níveis muito menores (GERSON 1985). FERES \& MORAES (1998) avaliaram 18 espécies vegetais em duas áreas florestais no Noroeste do Estado de São Paulo e encontraram 13 espécies de fitoseídeos. Outros estudos realizados em 33 pontos da Região Nordeste do Brasil, onde foram avaliadas 135 espécies vegetais, 38 espécies de ácaros predadores foram encontradas, sendo 32 espécies da família Phytoseiidae. As demais espécies pertenciam às famílias Ascidae, Cheyletidae, Cunaxidae, Erythraeidae e Stigmaeidae (MORAES et al. 1993).

Na cultura da macieira, E. brazilli tem sido citado como um dos agentes mais comuns no controle biológico de ácaros fitófagos no Rio Grande do Sul (LORENZATO 1987; LORENZATO \& SECCHI 1993). Na mesma cultura e também no Rio Grande 
do Sul, Ferla \& MORAES (1998) relataram maiores populações de E. alatus e $E$. brazilli. Segundo aqueles resultados e os resultados encontrados neste estudo, os ácaros do gênero Euseius são os predadores mais comuns na vegetação natural avaliada e na macieira sem tratamento fitossanitário, no Rio Grande do Sul. Devido ao aparente potencial apresentado por estas espécies como inimigos naturais de ácaros fitófagos, torna-se necessário desenvolver estudos futuros no sentido de definir sua importância nos agroecossistemas e na vegetação natural, assim como conhecer o potencial destas espécies em programas de controle biológico ou de manejo integrado de pragas.

\section{REFERÊNCIAS BIBLIOGRÁFICAS}

Aponte, O. \& J.A. McMurtry. 1995. Revision of the genus Iphiseiodes De Leon (Acari: Phytoseiidae). Internat. Jour. Acarol. 21 (3): 165-183.

AtHias-Henriot, C. 1977. Nouvelles notes sur les Amblyseiini. III. Sur le genre Cydnodromus:

Redefinition, compositions (Parasitiformes: Phytoseiidae). Entomophaga 22: 61-73.

BanKs, N. 1905. A treatise on the Acarina or mites. Proc. U.S. Nat. Mus. 28 (1382): 1-114.

ChAnT, D.A. 1955. Notes on mites of the genus Typhlodromus Scheuten, 1857 (Acarina: Laelapidae), with descriptions of the males of some species and the female of a new species. Canad. Entomol. 87 (11): 496-503.

. 1959. Phytoseiid mites (Acarina: Phytoseiidae). Part I. Bionomics of seven species in southeastern England. Part II. A taxonomic review of the family Phytoseiidae, with descriptions of thirthy-eight new species. Canad. Entomol. 91 (Suppl. 12): 1-166.

Chant, D.A. \& E.W. Baker. 1965. The Phytoseiidae (Acarina) of Central America. Mem. Entomol. Soc. Canada 41: 1-56.

Chant, D.A. \& J.A. McMurtry. 1994. A review of the subfamilies Phytoseinae and Typhlodrominae (Acari: Phytoseiidae). Internat. Jour. Acarol. 20 (4): 222-311.

Chant, D.A. \& E. YoshiDA-Shaul. 1984. A world review of the occidentalis species group in the genus Typhlodromus Scheuten (Acarina: Phytoseiidae). Can. Jour. Zool. 62: 1860-1871.

CHAUdHRI, W.M. 1968. Six new species of mites of the genus Amblyseius (Phytoseiidae) from Pakistan. Acarologia 10 (4): 550-562.

Daneshyar, H. \& H.A. Denmark. 1982. Phytoseiids of Iran (Acarina: Phytoseiidae). Internat. Jour. Acarol. 8: 3-14.

DeLeon, D. 1958. Four new Typhlodromus from southern Florida (Acarina: Phytoseiidae). Florida Entomol. 41: 73-76.

-1961. Eight new Amblyseius from Mexico with collection notes on two other species (Acarina: Phytoseiidae). Florida Entomol. 44 (2): 85-91.

.1962a. The cervices of some phytoseiid type specimens (Acarina: Phytoseiidae). Acarologia 4: $174-176$.

- 1962b. Twenty-three new Phytoseiidae, mostly from southeastern United States (Acarina: Phitoseiidae). Florida Entomol. 45 (1): 11-27.

1965a.Phytoseiid mites from Puerto Rico with descriptions of new species (Acarina: Mesostigmata), Florida Entomol. 48 (2): 121-131.

-1965b. A note on Neoseiulus Hughes, 1948 and new synonymy (Acarina: Phytoseiidae).

Proc. Entomol. Soc. Wash. 67 (1): 23.

-1966. Phytoseiidae of British Guyana with keys to species (Acarina: Mesostigmata). In:

Studies on the fauna of Suriname and other Guyanas 8: 81-102.

. 1967. Some mites of the Caribbean Area. Part I. Acarina on plants in Trinidad, West Indies. Lawrence, Allen Press Inc., 66p.

Denmark, H.A. 1966. Revision of the genus Phytoseius Ribaga, 1904 (Acarina: Phytoseiidae). Florida

Dept. Agr. Bull. 6: 1-105.

Revta bras. Zool. 19 (4): 1011 - 1031, 2002 
Denmark H.A. \& M.H. Muma. 1970. Some Phytoseiidae mites of Paraguay (Phytoseiidae: Acarina). Florida Entomol. 53 (4): 219-227.

$19-29$

1973. Phytoseiid mites of Brazil (Acarina: Phytoseiidae). Rev. Brasil. Biol. 33: 235-276.

1975. The Phytoseiidae (Acarina: Mesostigmata) of Puerto Rico. Jour. Agric. Univ. Puerto

Rico 59: 279-304.

1983. Revision of the genus Proprioseius Chant, 1957 (Acarina: Phytoseiidae). Florida Entomol. 49: 253-264.

1989. A revision of the genus Amblyseius Berlese, 1914 (Acari: Phytoseiidae). Occ. Papers Florida Sta. Coll. Arthropods 4: 1-149p.

Denmark, H.A.\& E. Schicha. 1983. Revision of the genus Phytoseiulus Evans (Acarina: Phytoseiidae).

Internat. Jour. Acarol. 9: 27-35.

Dosse, G. 1958. Die Spermathecae, ein zusatzliches Bestimmungsmerkmal bei Raubmilben (Acar.:Phytoseiidae), Pflanzenschutz Berichte, Amsterdam, 20 (1/2): 1-11.

EHARA, S. 1966. Some mites associated with plants in the state of São Paulo, Brazil, with a list of plant mites of South America. Jap. Jour. Zool. 15 (2): 129-150.

EHARA, S. \& G.J. DE MoRAES. 1998. A new species of Amblyseius (Euseius) (Acari: Phytoseiidae) from citrus in Uruguay. Entomol. Sci. 1 (1): 59-61.

El-Benhawy, E.M. 1975. New Amblyseius mites from Brasil. Rev. Brasil. Biol. 35: 549-552. 1979. Records on Phytoseiid (Acari) mites of Peru. Internat. Jour. Acarol. 5 (2): 111-116. 1984. Description of some phytoseiid mites from Brazil (Acarina: Phytoseiidae). Acarologia

25: $125-144$.

Evans, G.O. 1952. On a new predatory mite of economic importance. Bull. Entomol. Res. 43: 397-401.

Feres, R.J.F. \& G.J. DE MoraEs. 1998. Phytoseidae mites (Acari: Phytoseiidae) from woody areas in the State of São Paulo, Brazil. Sistem. Appl. Acarol. 3: 125-132.

FERLA, N.J. \&. G.J. DE Moraes. 1998. Ácaros predadores em pomares de maçã no Rio Grande do Sul. An. Soc. Entomol. Brasil 27 (4): 649-654.

Gerson, U. 1985. Other predaceous mites and spiders, p. 205-210. In: W. Helle \& M.W. SABelis (Eds).

Spider mites: Their biology, natural enemies and control 1B. Amsterdam, Elsevier, 458p.

Hoy, M.A.; Cunningham, G.L. \& L. KNutson. 1982. Biological control of pests by mites. Berkeley, Univ. California Press, 185p.

KEnNET, C.E. 1958. Some predaceous mites of the subfamilies Phytoseiinae and Aceosejinae (Acarina: Phytoseiidae, Aceosejidae) from central California with descriptions of new species. Ann. Entomol. Soc. Amer. 51: 471-479.

Kretter, S. \& G.J. DE Moraes. 1997. Phytoseiid mites (Acari: Phytoseiidae) from Guadaloupe and Martinique. Florida Entomol. 80 (3): 376-382.

LAING, J.E. \& N.F. KNOP. 1982. Potential uses of predaceous mites other than Phytoseiidae for biological control of orchard pests, p. 28-35. In: M.A. HoY; G.L. CunNingham \& L. KNUTSON (Eds). Biological Control of Pests by Mites. Berkeley, Univ. California, 185p.

Lorenzato, D. 1987. Controle biológico de ácaros fitófagos na cultura da macieira no município de Farroupilha, RS. Agronomia Sulriograndense, Porto Alegre, 23 (2): 167-183.

Lorenzato, D.; E.O. Grellmann; E.C. Chouéne \& L.M. Meyer-Cachapuz. 1986. Flutuação populacional de ácaros fitófagos e seus predadores associados à cultura da macieira (Malus domestica Bork) e efeitos dos controles químico e biológico. Agronomia Sulriograndense 22 (2): 215-242.

LoREnZATO, D. \& V.A. SECCHI. 1993. Controle biológico de ácaros da macieira no Rio Grande do Sul: 1 - Ocorrência e efeitos dos ácaros fitófagos e seus inimigos naturais em pomares submetidos ao controle biológico e com acaricidas. Rev. Brasil. Frutic., Cruz das Almas, 15 (1): 211-220.

McGregor, E.A. 1954. Two new mites in the genus Typhlodromus (Acarina: Phytoseiidae). Bull. S. Calif. Acad. Sci. 53: 89-92. 
MCMurtry, J.A. 1977. Some predaceous mites (Phytoseiidae) on citrus in the Mediterranean region. Entomophaga 22: 19-30.

- 1983. Phytoseiid mites from Guatemala, with descriptions of two new species and redefinitions of the genera Euseius, Typhloseiopsis and Typhlodromus occidentalis species group (Acari: Mesostigmata). Internat. Jour. Entomol. 25: 249-272.

MCMurtry, J.A. \& G.J. DE Moraes. 1984. Some phytoseiid mites from the South Pacific, with descriptions of new species and definition of the Amblyseius largoensis species group. Internat. Jour. Acarol. 10 (1): 27-37.

1989. Some phytoseiid mites from Peru with descriptions of four new species (Acari: Phytoseiidae). Internat. Jour. Acarol. 15 (3): 179-188.

Moraes, De G.J.; DE J.A. AlenCar; DE J.L.S. Lima; J.S. YanineK \& I. Delalibera JR. 1993. Alternative plant habitats for common phytoseiid predators of the cassava green mite (Acari: Phytoseiidae, Tetranychidae) in northeast Brazil. Experim. Appl. Acarol. 17: 77-90.

Moraes, G.J. DE \& N.C. MesA. 1988. Mites of the family Phytoseiidae (Acari) in Colombia, with descriptions of three new species. Internat. Jour. Acarol. 14: 71-88.

Moraes, G.J. DE; N.C. Mesa \& A. Braun. 1991. Some Phytoseiid mites of Latin America (Acari: Phytoseiidae). Internat. Jour. Acarol. 17 (2): 117-139.

Moraes, DE G.J. \& J.A. McMurtry. 1983. Phytoseiid mites (Acarina) of northeastern Brazil, with descriptions of four new species. Internat. Jour. Acarol. 9: 131-148.

Moraes, G.J. DE; J.A. McMurtry \& H.A. Denmark. 1986. A catalog of the mite family Phytoseiidae: references to taxonomy, synonymy, distribution and habitat. Brasília, EMBRAPA-DDT, 353p.

Moraes, G.J. DE \& J.V. DE OliveirA. 1982. Phytoseiidae mites of coastal Pernambuco in northeasthern Brazil. Acarologia 23 (4): 315-318.

Moraes, G.J. DE; N.C. Mesa; A. Braun \& E.L. Melo. 1994. Definition of the Amblyseius limonicus species group (Acari: Phytoseiidae), with descriptions of two new species and new records. Internat. Jour. Acarol. 20 (3): 209-217.

Muma, M.H. 1963. The genus Galendromus Muma, 1961 (Acarina: Phytoseiidae). Florida Entomol. 1: $15-41$.

Muma, M.H.; H.A. Denmark \& D. De Leon. 1970. Phytoseiidae of the Florida. Arthropods of Florida \& neighboring land areas. 6. Gainesville, Florida Dept. Agr. Cons. Serv. Div. Plant Ind., 150p.

NesbitT, H.H.J. 1951. A taxonomic study of the Phytoseinae (Family Laelapidae) Predaceous upon Tetranychidae of economic importance. Zool. Verhandel., Amsterdam, 12: 1-64.

SANTOS, M.A. \& J.E. LAING. 1985. Other predaceous mites and spiders, p.197-202. In: W. Helle \& M.W. SABELIS (Eds). Spider mites: their biology, natural enemies and control. 1B. Elsevier, Amsterdam, 458p.

Schultz, A.R. 1975. Os nomes científicos e populares das plantas do RS. Porto Alegre, Editora EMMA, 164p.

Schuster, R.O. \& A.E. Pritchard. 1963. Phytoseiid mites of California. Hilgardia, Berkeley, 34: 191-285.

Recebido em 24.VIII.2001; aceito em 01.X.2002. 